\title{
MODELING AGGREGATION AND GROWTH PROCESSES IN AN ALGAL POPULATION MODEL: ANALYSIS AND COMPUTATIONS
}

\author{
AZMY S. ACKLEH and BEN G. FITZPATRICK \\ Department of Mathematics and \\ Center for Research in Scientific Computation \\ North Carolina State University \\ Raleigh, NC 27695-8205
}

\begin{abstract}
Coagulation and cell division are both processes that are important in controlling the fate of phytoplankton cells through sedimentation. In this paper, we examine some basic mathematical issues associated with the development of a numerical simulation of an algal aggregation model. The numerical algorithm is then used to examine the basic model behavior.
\end{abstract}

\section{Introduction}

The formation of large aggregates of algal cells is fundamental to the dynamics of phytoplankton populations. The increase in mean volume of particles due to this process has the potential to limit phytoplankton population densities by increasing 
the flux to the bottom of the ocean. In order to understand this transfer of algal biomass from the ocean surface, one must examine those mechanisms in algae dynamics which lead to larger aggregates. In this paper, we are interested in two particular phenomena: coagulation and cell division. Coagulation, the formation of aggregates from smaller particles depends primarily on the tendency of the smaller particles to collide and stick together. General increase in aggregate mass (and, hence, volume) is also caused by division of the individual cells. Past models that have included cell division have typically assumed (Ackleh et al., 1994b) that the daughter cells do not remain in the aggregate - they instead enter the population as single cells. We consider in this paper a model that allows for some daughter cells to remain in the aggregate. The model equation for the size structured algal population is given by

$$
\left\{\begin{array}{l}
\frac{\partial \rho}{\partial t}+\frac{\partial(g \rho)}{\partial x}=F(\rho) \\
g\left(x_{0}\right) \rho\left(t, x_{0}\right)=K(\rho) \\
\rho(0, x)=\rho_{0}(x)
\end{array}\right.
$$

in which

$$
\begin{aligned}
& F(\rho)=\frac{1}{2} \int_{x_{0}}^{x-x_{0}} \beta(x-y, y) \rho(t, x-y) \rho(t, y) d y \\
& -\rho(t, x) \int_{x_{0}}^{x_{1}} \beta(x, y) \rho(t, y) d y-\frac{w \rho}{Z},
\end{aligned}
$$

and,

$$
K(\rho)=\int_{x_{0}}^{x_{1}} q(x) \rho(t, x) d x
$$

Here the $x$ variable represents the size $\left(\mathrm{mm}^{3}\right)$ of the aggregate, and the $t$ variable represents time. The function $\rho(t, x)$ is the density of aggregates of size $x$ 
at time $t$. The function $g=g(x)$ is the size-dependent growth rate, which models the increase in an aggregates size due to cell reproduction, $w=w(x)$ is the sizedependent sinking rate and $Z$ is the depth of the surface layer. The term $\frac{w \rho}{Z}$ in the function $F(\rho)$ represents the loss of particles of size $x$ due to sinking out of the surface layer. The "stickiness function" $\beta(x, y)$ represents the likelihood of an aggregate of size $x$ sticking to an aggregate of size $y$. The stickiness function includes several effects; it is usually taken (see, e.g., Ackleh et. al, 1994a) to be of the form

$$
\beta(x, y)=c_{1}\left(d_{x}+d_{y}\right)^{3} E C_{x, y}+(\pi / 4) *\left(d_{x}+d_{y}\right)^{2}\left|w_{x}-w_{y}\right| E C_{x, y}
$$

The first term models collisions due to horizontal shear flow in the surface layer, while the second models collisions due to differences in settling rates. The factor $E C$ is the contact efficiency, the probability that particles in close proximity actually come in contact. The factor $c_{1}$ is a constant involving the viscosity of the water, $d_{x}$ is the diameter of an aggregate of size $x$, and $w_{x}$ is the settling rate of an aggregate of size $x$. The first term in $F(\rho)$ expresses the rate at which collisions occur to form new particles with the size $x$ and $x+d x$ while the second term expresses the rate at which collisions cause particles to be lost from the same interval. The function $q(x)$ represents the number of new cells that fall off an aggregate of size $x$ and join the single cell population and $K(\rho)$ is a function that describes the addition of new cells to the population of single cells.

In order to use such a model as a predictive tool, one must understand the basic behavior of its solutions. Toward that end, in Section 2, we examine existence, uniqueness, and regularity questions for solutions of (1.1). In order to compare 
growth and coagulation processes in the model, we construct and analyze a numerical approximation scheme. Section 3 contains the numerical analysis, and in Section 4, we present computational results which illustrate the effects of aggregate growth and coagulation.

\section{Existence, Uniqueness and Regularity of Solutions}

We begin our investigation of the model by examining basic questions of wellposedness. We shall use the space $H=L^{2}\left(x_{0}, x_{1}\right)$, and use the following abstract form of equation (1.1)

$$
\dot{\rho}=A \rho+F(\rho)
$$

where $F$ is defined in (1.2), and the linear operator $A: \operatorname{dom} A \subset H \rightarrow H$ given by

$$
A \phi=-\frac{\partial}{\partial x}(g \phi)
$$

with

$$
\begin{aligned}
& \qquad \begin{array}{l}
\operatorname{dom} A=\left\{\phi \in H \mid g \phi \in H^{1}\left(x_{0}, x_{1}\right), \lim _{x \rightarrow x_{1}}(g \phi)(x)=0, g \phi\left(x_{0}\right)=K(\phi)\right\} \\
\text { where } K(\phi)=\int_{x_{0}}^{x_{1}} q(x) \phi(t, x) d x .
\end{array}
\end{aligned}
$$


We make the following assumptions about the model parameters in order to establish existence and uniqueness. These conditions have been used in other structured population problems (Banks Kappel, 1991; Ackleh et.al., 1994).

(H1) $g \in W^{1, \infty}\left(x_{0}, x_{1}\right), g(x)>0$ for $x_{0} \leq x<x_{1}$ and $g\left(x_{1}\right)=0$

(H2) $w \in L^{\infty}\left(x_{0}, x_{1}\right)$ and $w \geq 0$ a.e on $\left[x_{0}, x_{1}\right]$

(H3) $q \geq 0$ a.e on $\left[x_{0}, x_{1}\right]$ and $q \in L^{\infty}\left(x_{0}, x_{1}\right)$

(H4) $\beta \in L^{\infty}\left(\left[x_{0}, x_{1}\right] \times\left[x_{0}, x_{1}\right]\right), \beta(x, y)=\beta(y, x)$ and

$$
\left\{\begin{array}{l}
\beta(x, y) \geq 0 \text { if } x+y \leq x_{1} \\
\beta(x, y)=0 \text { if } x+y>x_{1}
\end{array}\right.
$$

We have from Banks et al. (1991) that $A$ is an infinitesimal generator for a $C_{0}$-semigroup $T(t)$ that satisfies $\|T(t)\| \leq e^{\omega t}$.

Using the semigroup, we consider the mild form of the equation, given by

$$
\rho(t)=T(t) \rho_{0}+\int_{0}^{t} T(t-s) F(\rho(s)) d s,
$$

which provides the first step in solving (1.1).

Lemma 2.1. For $\rho_{0} \in H,(1.1)$ has a unique mild solution $\rho(t, x)$ on $\left[0, t_{\max }\right)$ for some $t_{\max }>0$.

proof.

Since $F$ is independent of $t$, we need only to show (Pazy, theorem 1.4, page 185) that $F: H \rightarrow H$ and that $F$ is locally lipschitz. First we show that $F: H \rightarrow H$. From (H4) we have that $\|\beta\|_{\infty} \leq B$ for some constant $B$.

Consider the first integral term in the function $F(\rho)$. We have 


$$
\begin{aligned}
\frac{1}{2}\left|\int_{x_{0}}^{x-x_{0}} \beta(x-y, y) \rho(x-y) \rho(y) d y\right| & \leq \frac{1}{2} B \int_{x_{0}}^{x-x_{0}}|\rho(x-y) \rho(y)| d y \\
& \leq \frac{1}{2} B\left(\int_{x_{0}}^{x-x_{0}}|\rho(x-y)|^{2} d y\right)^{\frac{1}{2}}\left(\int_{x_{0}}^{x-x_{0}}|\rho(y)|^{2} d y\right)^{\frac{1}{2}} \\
& \leq \frac{1}{2} B \|\left.\rho\right|^{2}
\end{aligned}
$$

Hence

$$
\frac{1}{2} \int_{x_{0}}^{x-x_{0}}\left|\int_{x_{0}}^{x-x_{0}} \beta(x-y, y) \rho(x-y) \rho(y) d y\right|^{2} \leq\left(\frac{1}{2} B\right)^{2} \|\left.\rho\right|^{4}\left(x_{1}-x_{0}\right)
$$

In a similar manner one can find a bound on the second term of $F(\rho)$. Hence we get that

$$
\|F(\rho)\| \leq \frac{3}{2} B\|\rho\|^{2}\left(x_{1}-x_{0}\right)^{\frac{1}{2}}+\frac{\|w\|_{\infty}}{Z}\|\rho\|
$$

To show that $F$ is locally Lipschitz, we let $u, v \in H$ with $\|u\| \leq C$ and $\|v\| \leq C$ we want to show that

$$
\|F(u)-F(v)\| \leq L(C) \mid u-v \|
$$

Again we start by considering the first term of $F(\rho)$ :

$$
\begin{aligned}
\mid \int_{x_{0}}^{x-x_{0}} \beta(x-y, y)(u(x-y) u(y) & -v(x-y) v(y)) d y \mid \\
\leq & B \int_{x_{0}}^{x-x_{0}}(|u(x-y) u(y)-u(x-y) v(y)| \\
& +|u(x-y) v(y)-v(x-y) v(y)|) d y
\end{aligned}
$$




$$
\begin{aligned}
& \leq B\left(\int_{x_{0}}^{x-x_{0}}|u(x-y)|^{2} d y\right)^{\frac{1}{2}}\left(\int_{x_{0}}^{x-x_{0}}|u(y)-v(y)|^{2} d y\right)^{\frac{1}{2}} \\
& +B\left(\int_{x_{0}}^{x-x_{0}}|v(y)|^{2} d y\right)^{\frac{1}{2}}\left(\int_{x_{0}}^{x-x_{0}}|u(x-y)-v(x-y)|^{2} d y\right)^{\frac{1}{2}} \\
& \leq B(\|u\|+\|v\|)\|u-v\| .
\end{aligned}
$$

One can obtain a similar bound for the second term of $F(\rho)$ and hence we can show that

$$
\begin{aligned}
\|F(u)-F(v)\| & \leq \frac{3}{2}\left(x_{1}-x_{0}\right)^{\frac{1}{2}} B(\|u\|+\|v\|)\|u-v\| \\
& \leq 3\left(x_{1}-x_{0}\right)^{\frac{1}{2}} C \mid u-v\left\|+\frac{\|w\|_{\infty}}{Z}\right\| u-v\|=L(C)\| u-v \|
\end{aligned}
$$

Hence $F(\rho)$ is locally Lipschitz and this establishes the desired result.

Lemma 2.2. If $\rho_{0} \in \operatorname{dom} A$, then the unique mild solution is a classical one.

\section{Proof.}

It is enough to show that $F: H \rightarrow H$ is continuously differentiable from $H \rightarrow H$ (see Pazy, Theorem 1.5, page 187). Let $u \in H$ we want to show that

$$
F(u+h)-F(u)=L(u, h)+O(u, h)
$$

for $h \in H$, where $L(u,.) \in \mathcal{L}(H)$, the map $u \rightarrow L(u, \cdot)$ is continuous in the uniform operator topology, and that

$$
\lim _{\|h\| \rightarrow 0} \frac{\|O(u, h)\|}{\|h\|}=0
$$

First we note that 


$$
\begin{aligned}
F(u+h)-F(u)= & \frac{1}{2} \int_{x_{0}}^{x-x_{0}} \beta(x-y, y)(h(x-y) u(y)+u(x-y) h(y)) d y \\
& -u(x) \int_{x_{0}}^{x_{1}} \beta(x, y) h(y) d y+h(x) \int_{x_{0}}^{x_{1}} \beta(x, y) u(y) d y-\frac{w h(x)}{Z} \\
& +\frac{1}{2} \int_{x_{0}}^{x-x_{0}} \beta(x-y, y) h(x-y) h(y) d y \\
& -h(x) \int_{x_{0}}^{x_{1}} \beta(x, y) h(y) d y=L(u, h)+O(u, h) .
\end{aligned}
$$

where

$$
\begin{aligned}
O(u, h)= & \frac{1}{2} \int_{x_{0}}^{x-x_{0}} \beta(x-y, y) h(x-y) h(y) d y \\
& -h(x) \int_{x_{0}}^{x_{1}} \beta(x, y) h(y) d y
\end{aligned}
$$

For $h \in H$ we have shown earlier that

$$
\|O(u, h)\| \leq \frac{3}{2} B\|h\|^{2}\left(x_{1}-x_{0}\right)^{\frac{1}{2}}
$$

hence it is clear that

$$
\lim _{\|h\| \rightarrow 0} \frac{\|O(u, h)\|}{\|h\|}=0 .
$$

Linearity of the operator $L$ is clear, thus, it is left to show that the map $u \rightarrow L(u, \cdot)$ is continuous in the uniform topology. Let $u_{n} \rightarrow u$ in $H$. Then

$$
\begin{aligned}
\left|L\left(u_{n}, h\right)-L(u, h)\right|= & \mid \frac{1}{2} \int_{x_{0}}^{x-x_{0}} \beta(x-y, y)\left(h(x-y)\left(u_{n}(y)-u(y)\right)\right. \\
& \left.+\left(u_{n}(x-y)-u(x-y)\right) h(y)\right) d y \\
& -\left(u_{n}(x)-u(x)\right) \int_{x_{0}}^{x_{1}} \beta(x, y) h(y) d y
\end{aligned}
$$




$$
\begin{aligned}
& +h(x) \int_{x_{0}}^{x_{1}} \beta(x, y)\left(u_{n}(y)-u(y)\right) d y \mid \\
\leq & B\left\|u_{n}-u\right\|\|h\|+2 B\left\|u_{n}-u\right\|\|h\|
\end{aligned}
$$

Hence we have that

$$
\left\|L\left(u_{n}, h\right)-L(u, h)\right\| \leq(3 B)^{2}\left\|u_{n}-u\right\|\|h\|\left(x_{1}-x_{0}\right)
$$

Hence

$$
\| L\left(u_{n}, .-L(u, .)\left\|_{\mathcal{L}(H)}=\sup _{\|h\| \leq 1}\right\| L\left(u_{n}, h\right)-L(u, h) \| \rightarrow 0\right.
$$

Theorem 2.1. If $\rho_{0} \in \operatorname{dom} A$ satisfies $\rho_{0} \geq 0 \forall x \in\left[x_{0}, x_{1}\right)$, then the local solution of (1.1) is a global solution.

Proof. The basic idea in this argument is finding an upper bound for the solution, so that the maximum interval of existence is of infinite length. Notice first the positive quadrant of $R^{2} \times\left[0, t_{\max }\right)$ is invariant; i.e, $\rho(t, x) \geq 0$ a.e. for all $t \in\left[0, t_{\max }\right)$. Define $H(t)=\int_{x_{0}}^{x_{1}} x \rho d x$. From (1.1), we obtain

$$
\frac{d}{d t} \int_{x_{0}}^{x_{1}} x \rho d x=-\int_{x_{0}}^{x_{1}} x(g \rho)_{x} d x-\int_{x_{0}}^{x_{1}} x \frac{w \rho}{Z} d x+\int_{x_{0}}^{x_{1}} x F(\rho) d x
$$

A tedious but straightforward calculation (see Ackleh et.al., 1994) shows that

$$
\int_{x_{0}}^{x_{1}} x F(\rho) d x=0
$$

Then,

$$
\begin{aligned}
\frac{d H}{d t} & =\int_{x_{0}}^{x_{1}}-\frac{w \rho}{Z} d x-\int_{x_{0}}^{x_{1}} x(g \rho)_{x} d x \\
& \leq \frac{\|w\|_{\infty}}{z} \int_{x_{0}}^{x_{1}} x \rho d x+\int_{x_{0}}^{x_{1}} g \rho d x-\left.x g \rho\right|_{x_{0}} ^{x_{1}} \\
& \leq \frac{\|w\|_{\infty}}{z} H(t)+\frac{\|g\|_{\infty}}{x_{0}} H(t)+\|q\|_{\infty} H(t)
\end{aligned}
$$


Hence define $E=\frac{\|w\|_{\infty}}{z}+\frac{\|g\|_{\infty}}{x_{0}}+\|q\|_{\infty}$ then we have

$$
\frac{d H}{d t} \leq E H(t)
$$

This implies that

$$
H(t) \leq e^{E t+c_{1}}
$$

for some constant $c_{1}$. But then we have that,

$$
\int_{x_{0}}^{x_{1}} \rho d x \leq \frac{1}{x_{0}} H(t)<e^{E T+c_{1}}<\infty
$$

Now since $\rho$ is classical solution hence absolutely continuous, we have for any compact set $[0, \mathrm{~T}]$ :

$$
\sup _{t \in[0, T]} \sup _{x \in\left[x_{0}, x_{1}\right]}|\rho(t, x)|<\infty
$$

then (Pazy theorem 1.4, page 185) this implies that $t_{\max }=\infty$.

\section{A Numerical Approximation Scheme}

For our approximation scheme we use an approach similar to that used by Banks and Kappel (1989). In addition to the assumption (H1)-(H4) we assume the following assumptions throughout the rest of the paper:

(H5) $g$ is contiuously differentiable on $\left[x_{0}, x_{1}\right]$.

(H6) $w$ is continuous on $\left[x_{0}, x_{1}\right]$.

(H7) $q$ is continuous on $\left[x_{0}, x_{1}\right]$. 
For $N=1,2, \cdots$ and $j=0, \cdots, N-1$ let $x_{j}=x_{0}+\frac{j\left(x_{1}-x_{0}\right)}{N}$ and define

$$
\phi_{j}^{N}=\left\{\begin{array}{l}
1 \text { for } x_{j} \leq x<x_{j+1} \\
0 \text { elsewhere }
\end{array}\right.
$$

For each $N$, these functions form an orthogonal basis for the subspaces

$$
X^{N}=\left\{f \in H \mid f=\sum_{j=0}^{N-1} f_{j} \phi_{j}^{N}, f_{j} \in R\right\}
$$

Define the projections $\pi^{N}: H \rightarrow X^{N}$ by

$$
\pi^{N} f=\sum_{j=0}^{N-1} f_{j} \phi_{j}^{N} \text { with } f_{j}=N \int_{x_{j}}^{x_{j+1}} f(x) d x, \quad j=0, \cdots, N-1
$$

Straightforward calculations show that $\pi^{N}$ is the orthogonal projection of $H$ onto $X^{N}$.

We define the approximating generators $A^{N}: X^{N} \rightarrow X^{N}$ of the infinitesimal generator $A$ by:

$$
\left(A^{N} f\right)(x)=-\sum_{j=1}^{N-1} N\left(g\left(x_{j+1}\right) f_{j}-g\left(x_{j}\right) f_{j-1}\right) \phi_{j}^{N}(x)+\sum_{j=0}^{N-1} q\left(x_{j+1}\right) f_{j} \phi_{0}^{N}(x),
$$

for $f \in X^{N}$.

Applying $A^{N}$ to the basis elements we get:

$$
A^{N} \phi_{k}^{N}=-N g\left(x_{k+1}\right) \phi_{k}^{N}+N g\left(x_{k+1}\right) \phi_{k+1}^{N}+q\left(x_{k+1}\right) \phi_{0}^{N}
$$

Hence our matrix representation $\left[A^{N}\right]$ of $A^{N}$ with respect to our basis is given by 


$$
\left(\begin{array}{ccccc}
q\left(x_{1}\right)-N g\left(x_{1}\right) & q\left(x_{2}\right) & q\left(x_{3}\right) & \ldots & q\left(x_{N}\right) \\
N g\left(x_{1}\right) & -N g\left(x_{2}\right) & 0 & \ldots & 0 \\
0 & N g\left(x_{2}\right) & -N g\left(x_{3}\right) & \ldots & 0 \\
\ldots \ldots \ldots \ldots \ldots \ldots \ldots \ldots \ldots \ldots \ldots \ldots \ldots \ldots \ldots \ldots \ldots \ldots \ldots \ldots \ldots \ldots \ldots & \ldots \ldots \ldots
\end{array}\right)
$$

Thus, our approximating problem of (1.1) can be stated in following simple operator form:

$$
\left\{\begin{array}{l}
\frac{\partial \rho^{N}}{\partial t}=A^{N} \rho^{N}+\pi^{N} F\left(\rho^{N}\right) \\
\rho^{N}(0, x)=\pi^{N} \rho_{0}(x) .
\end{array}\right.
$$

To prove convergence of the scheme we will use the Trotter-Kato approach (in particular, we shall use the pi-convergence form as given in Banks and Kunisch, Theorem 1.14, page 40), which involves stability (uniform boundedness of the approximating semigroups) and consistency (convergence of the approximating generators). First we will prove stability of the scheme. Let $T^{N}(t)$ be the semigroups on $X^{N}$ defined by

$$
T^{N}(t)=e^{A^{N} t}
$$

Lemma 3.1. $\left\|T^{N}(t)\right\| \leq e^{\|q\|_{\infty} t}, \forall N$.

\section{Proof.}

For any $f \in X^{N}$ we have that:

$$
\|f\|_{L^{2}}^{2}=\frac{1}{N} \sum_{j=0}^{N-1}\left|f_{j}\right|^{2} .
$$


Hence $X^{N}$ is a subspace of $H$ which is isometrically isomorphic to $R^{N}$, when equipped with the norm $\|\cdot\|_{N}=\frac{1}{N} \sum_{j=0}^{N-1}\left|f_{j}\right|^{2}$. Corresponding to this is norm is the is the usual two matrix norm denoted by $\|B\|_{2}$ for $B \in R^{N \times N}$

If $B$ is an upper Hessenberg matrix, then its eigenvalues are of geometric multiplicity of one; hence, it may be diagonalized, and $\left\|e^{B t}\right\|_{2} \leq e^{\sigma t}$, where

$$
\sigma=\max \{\operatorname{Re}(\lambda): \lambda \text { is an eigenvalue of } B\}
$$

Since our matrix $\left[A^{N}\right]$ is in upper Hessenberg form we only need to establish an upper bound on the eigenvalues. To do so we use Gershgorin disks to get the following estimate:

$$
\left|\lambda+N g\left(x_{i}\right)\right| \leq q\left(x_{i}\right)+N g\left(x_{i}\right)
$$

Hence this implies that $\operatorname{Re}(\lambda) \leq\|q\|_{\infty}$, so that

$$
\left\|e^{A^{N} t}\right\|_{2} \leq e^{t\|q\| \infty}
$$

which establishes the result.

Our next task is to prove that the approximating generators converge on a "sufficiently big" subset of $L^{2}\left(x_{0}, x_{1}\right)$, We begin with the following lemma.

Lemma 3.2. The set $D=\left\{f \mid f \in \operatorname{dom} A, f \in C^{1}\left[x_{0}, x_{1}\right]\right\}$ is dense in $H$.

\section{Proof.}

Clearly $S=\left\{f \mid f \in C^{1}\left[x_{0}, x_{1}\right]\right.$ supp $\left.f \subset[0,1)\right\}$ is dense in H. We will argue 
that $D$ is dense in $S$ to establish the result. Let $f \in S$ and for each $\epsilon>0$ define

$$
f_{\epsilon}(x)=\left\{\begin{array}{l}
\left(\frac{f^{\prime}\left(x_{0}+\epsilon\right)}{\epsilon}-\frac{f\left(x_{0}+\epsilon\right)-\gamma_{\epsilon}}{\epsilon^{2}}\right)\left(x-x_{0}\right)^{2} \\
+\left(2 \frac{f\left(x_{0}+\epsilon\right)-\gamma_{\epsilon}}{\epsilon}-f^{\prime}(\epsilon)\right)\left(x-x_{0}\right)+\gamma_{\epsilon} \quad x_{0} \leq x<x_{0}+\epsilon \\
f(x) \quad x_{0}+\epsilon \leq x<x_{1}
\end{array}\right.
$$

Then clearly $f_{\epsilon}(x)$ is continuously differentiable and $\left(g f_{\epsilon}\right)^{\prime} \in H$. Since supp $f \subset\left[x_{0}, x_{1}\right)$ then let $\delta>x_{0}$ s.t. $\operatorname{supp} f \in\left[x_{0}, \delta\right]$ this implies that $\operatorname{supp} f_{\epsilon} \subset\left[x_{0}, \delta\right]$. Hence $f_{\epsilon}\left(x_{1}\right)=0$ and $g\left(x_{1}\right)=0$ so $\lim _{\xi \rightarrow x_{1}}\left(g f_{\epsilon}\right)(\xi)=0$. It remains to show that $\gamma_{\epsilon}$ can be chosen such that $\left(g f_{\epsilon}\right)\left(x_{0}\right)=K\left(f_{\epsilon}\right)$ and that $f_{\epsilon} \rightarrow f$ in $H$. Note that $f_{\epsilon}\left(x_{0}\right)=\gamma_{\epsilon}$. For this equality to hold, we must have

$$
\begin{aligned}
\gamma_{\epsilon} g\left(x_{0}\right)= & \int_{x_{0}}^{x_{0}+\epsilon} q(x)\left(\gamma_{\epsilon}-2 \frac{\gamma_{\epsilon}}{\epsilon}\left(x-x_{0}\right)+\frac{\gamma_{\epsilon}}{\epsilon^{2}}\left(x-x_{0}\right)^{2}\right) d x \\
& +\int_{x_{0}}^{x_{0}+\epsilon} q(x)\left(-\frac{f\left(x_{0}+\epsilon\right)}{\epsilon^{2}}\left(x-x_{0}\right)^{2}+\frac{f^{\prime}\left(x_{0}+\epsilon\right)}{\epsilon}\left(x-x_{0}\right)^{2}\right. \\
& \left.-2 \frac{f\left(x_{0}+\epsilon\right)}{\epsilon}\left(x-x_{0}\right)-f^{\prime}\left(x_{0}+\epsilon\right) x\right) d x \\
& +\int_{x_{0}+\epsilon}^{x_{1}} q(x) f(x) d x,
\end{aligned}
$$

or

$$
\begin{aligned}
\gamma_{\epsilon}\left(g\left(x_{0}\right)-\right. & \left.\int_{x_{0}}^{x_{0}+\epsilon} q(x)\left(1-2 \frac{x}{\epsilon}+\frac{x^{2}}{\epsilon^{2}}\right) d x\right)= \\
& \int_{x_{0}}^{x_{0}+\epsilon} q(x)\left(\left(x-x_{0}\right)^{2}\left(\frac{f^{\prime}\left(x_{0}+\epsilon\right)}{\epsilon}-\frac{f\left(x_{0}+\epsilon\right)}{\epsilon^{2}}\right)\right. \\
& +\left(x-x_{0}\right)\left(2 \frac{f\left(x_{0}+\epsilon\right)}{\epsilon}-f^{\prime}\left(x_{0}+\epsilon\right)\right) d x+\int_{x_{0}+\epsilon}^{x_{1}} q(x) f(x) d x
\end{aligned}
$$


Since $g\left(x_{0}\right)>0$ and $\int_{x_{0}}^{x_{0}+\epsilon} q(x)\left(1-2 \frac{x-x_{0}}{\epsilon}+\frac{\left(x-x_{0}\right)^{2}}{\epsilon^{2}}\right) d x \rightarrow 0$ as $\epsilon \rightarrow 0$, we can choose

$$
\begin{aligned}
\gamma_{\epsilon}= & {\left[g\left(x_{0}\right)-\int_{x_{0}}^{x_{0}+\epsilon} q(x)\left(1-2 \frac{x-x_{0}}{\epsilon}+\frac{\left(x-x_{0}\right)^{2}}{\epsilon^{2}}\right) d x\right]^{-1} } \\
& \left(\int _ { x _ { 0 } } ^ { x _ { 0 } + \epsilon } q ( x ) \left(\left(x-x_{0}\right)^{2}\left(\frac{f^{\prime}\left(x_{0}+\epsilon\right)}{\epsilon}-\frac{f\left(x_{0}+\epsilon\right)}{\epsilon^{2}}\right)\right.\right. \\
& +\left(x-x_{0}\right)\left(2 \frac{f\left(x_{0}+\epsilon\right)}{\epsilon}-f^{\prime}\left(x_{0}+\epsilon\right)\right) d x \\
& \left.+\int_{x_{0}+\epsilon}^{x_{1}} q(x) f(x) d x\right)
\end{aligned}
$$

But as $\epsilon \rightarrow 0 \gamma_{\epsilon} \rightarrow g\left(x_{0}\right)^{-1} K(f)$ hence we have that $f_{\epsilon} \rightarrow f$, which gives us the desired result.

A property of $D$ that will be needed to apply Trotter-Kato is given in the following lemma.

Lemma 3.3. There exist a $\lambda_{0}$ with $\operatorname{Re} \lambda_{0}>\omega$ such that $\overline{\left(\lambda_{0}-A\right) D}=H$.

\section{Proof.}

Since $S=\left\{f \mid f \in C^{1}\left[x_{0}, x_{1}\right] \operatorname{supp} f \subset[0,1)\right\}$ is dense in $H$, it is enough to show that $S \subset(\lambda-A) D$. Let $\phi \in S$. Then

$$
\lambda \psi+(g \psi)^{\prime}=\phi
$$

is equivalent to

$$
\psi(x)=\psi\left(x_{0}\right) \frac{g\left(x_{0}\right)}{g(x)} e^{-\int_{x_{0}}^{x} \frac{\lambda}{g(\sigma)} d \sigma}+\int_{x_{0}}^{x} \frac{\phi(\tau)}{g(x)} e^{-\int_{\tau}^{x} \frac{\lambda}{g(\sigma)} d \sigma} d \tau
$$


Easy calculations show that $\psi^{\prime} \in H$. Hence, since $g(x) \in C^{1}\left[x_{0}, x_{1}\right]$, we have that $(g \psi)^{\prime} \in H$.

Now multiplying (3.1) by $g(x)$, we obtain,

$$
g(x) \psi(x)=\psi\left(x_{0}\right) g\left(x_{0}\right) e^{-\int_{x_{0}}^{x} \frac{\lambda}{g(\sigma)} d \sigma}+\int_{x_{0}}^{x} \phi(\tau) e^{-\int_{\tau}^{x} \frac{\lambda}{g(\sigma)} d \sigma} d \tau
$$

Now, by dominated convergence theorem, we have that

$$
\lim _{x \rightarrow x_{1}} g(x) \psi(x)=0
$$

Hence it remains to show that there a $\lambda_{0}$ with $\operatorname{Re} \lambda_{0}>w$ such that $g\left(x_{0}\right) \psi\left(x_{0}\right)=$ $K(\psi)$. Toward that end, we define

$$
B\left(x_{0}\right)=\int_{x_{0}}^{x} \frac{\lambda}{g(\sigma)} d \sigma
$$

Then using the boundary condition $g\left(x_{0}\right) \psi\left(x_{0}\right)=K(\psi)$ we get

$$
\begin{aligned}
g\left(x_{0}\right) \psi\left(x_{0}\right) & =\int_{x_{0}}^{x_{1}} q(x)\left(\frac{\psi\left(x_{0}\right) g\left(x_{0}\right)}{g(x)} e^{-B\left(x_{0}\right)}\right)+\int_{x_{0}}^{x} \frac{\phi(\tau)}{g(x)} e^{-B\left(x_{0}\right)} d \tau \\
& =g\left(x_{0}\right) \psi\left(x_{0}\right) K\left(\frac{1}{g(x)} e^{-B\left(x_{0}\right)}+K\left(\frac{1}{g(x)} \int_{x_{0}}^{x} \phi(\tau) e^{-B(\tau)} d \tau\right)\right.
\end{aligned}
$$

hence we get that :

$$
\left(1-K\left(\frac{1}{g} \exp \left(-\int_{x_{0}} \frac{\lambda}{g(\sigma)} d \sigma\right)\right)\right) \psi\left(x_{0}\right) g\left(x_{0}\right)=K\left(\frac{1}{g} \int_{x_{0}} \phi(\tau) e^{-B(\tau)} d \tau\right)
$$

Next, we note that

$$
\frac{1}{g(x)} \exp \left(-\int_{x_{0}}^{x} \frac{\lambda}{g(\sigma)} d \sigma\right)=\frac{1}{g\left(x_{0}\right)} \exp \left(-\int_{x_{0}}^{x} \frac{\lambda+g^{\prime}(\sigma)}{g(\sigma)} d \sigma\right)
$$


hence, using (H1), we see that we can choose $R e \lambda>\omega$ large enough such that (see Banks and Kappel, 1991),

$$
\left|K\left(\frac{1}{g} \exp \left(-\int_{x_{0}} \frac{\lambda}{g(\sigma)} d \sigma\right)\right)\right|<1 .
$$

Lemma 3.4 For each $y \in D,\left\|A^{N} \pi^{N} y-\pi^{N} A y\right\|_{N} \rightarrow 0$ as $N \rightarrow \infty$ proof.

$$
\pi^{N} A y=\sum_{i=0}^{N-1}\left(N \int_{x_{k}}^{x^{k+1}}-(g y)^{\prime} d x \phi_{k}^{N}=-\sum_{i=0}^{N-1} N\left(g\left(x_{k+1}\right) y_{k+1}-g\left(x_{k}\right) y_{k}\right) \phi_{k}^{N}\right.
$$

and

$$
\pi^{N} y=\sum_{k=0}^{N-1}\left(N \int_{x_{k}}^{x_{k+1}} y(\xi) d \xi\right) \phi_{k}^{N}=\sum_{k=0}^{N-1} \alpha_{k} \phi_{k}^{N}
$$

with

$$
\alpha_{k}=N \int_{x_{k}}^{x_{k+1}} y(\xi) d \xi
$$

But

$$
A^{N} \phi_{k}^{N}=-N g\left(x_{k+1}\right) \phi_{k}^{N}+N g\left(x_{k+1}\right) \phi_{k+1}^{N}+q\left(x_{k}\right) \phi_{0}^{N}
$$

Hence :

$$
\begin{aligned}
\left\|\pi^{N} A y-A^{N} \pi^{N} y\right\|_{N}^{2}= & \frac{1}{N} \sum_{i=0}^{N-1}\left|\left(\pi^{N} A y\right)_{i}-\left(A^{N} \pi^{N} y\right)_{i}\right|^{2} \\
= & \frac{1}{N}\left(\left|-\alpha_{0} N g\left(x_{1}\right)+\sum_{k=0}^{N-1} \alpha_{k} q\left(x_{k+1}\right)+N g\left(x_{1}\right) y_{1}-N g\left(x_{0}\right) y_{0}\right|^{2}\right. \\
& +\sum_{i=1}^{N-1} \mid-N g\left(x_{k+1}\right) y_{k+1}+N g\left(x_{k}\right) y_{k}+N \alpha_{k} g\left(x_{k+1}\right)
\end{aligned}
$$




$$
\begin{aligned}
& \left.-\left.N \alpha_{k-1} g\left(x_{k}\right)\right|^{2}\right) \\
= & \frac{1}{N}\left(\left|-N g\left(x_{1}\right) y_{1}+N g\left(x_{0}\right) y_{0}+N \alpha_{0} g\left(x_{1}\right)-\sum_{k=0}^{N-1} \alpha_{k} q\left(x_{k+1}\right)\right|^{2}\right. \\
& +\sum_{i=1}^{N-1} \mid-N g\left(x_{k+1}\right) y_{k+1}+N g\left(x_{k}\right) y_{k}+N^{2} \int_{x_{k}}^{x_{k+1}} y(x) d x g\left(x_{k+1}\right) \\
& \left.-\left.N^{2} \int_{x_{k-1}}^{x_{k}} y(x) d x g\left(x_{k}\right)\right|^{2}\right) \\
= & \frac{1}{N}\left(\mid-N g\left(x_{1}\right) y_{1}+N g\left(x_{0}\right) y_{0}+N^{2} \int_{x_{0}}^{x_{1}} y(x) d x g\left(x_{1}\right)\right. \\
& -\left.\sum_{k=0}^{N-1} \alpha_{k} q\left(x_{k+1}\right)\right|^{2}+\sum_{i=0}^{N-1} \mid-N g\left(x_{k+1}\right) y_{k+1}+N g\left(x_{k}\right) y_{k}+ \\
& N\left(y_{k+1}-\frac{1}{2 N} y^{\prime}(\xi) g\left(x_{k+1}\right)-N\left(y_{k}-\left.\frac{1}{2 N} y^{\prime}(\bar{\xi}) g\left(x_{k}\right)\right|^{2}\right)\right. \\
\leq & \frac{1}{N}\left(\left|N g\left(x_{0}\right) y_{0}-\sum_{k=0}^{N-1} \alpha_{k} q\left(x_{k+1}\right)+\frac{1}{2} y^{\prime}(\hat{\xi}) g\left(x_{1}\right)\right|^{2}\right. \\
& +\sum_{i=1}^{N-1}\left\{|| y^{\prime}|| \mid g\left(x_{k+1}-g\left(x_{k}\right)|+||g \| \infty| y^{\prime}(\xi)-y^{\prime}(\bar{\xi}) \mid\right\}\right.
\end{aligned}
$$

Hence as $N \rightarrow \infty$ then the right hand side $\rightarrow 0$ by the uniform continuity of $y^{\prime}$ and $g$.

Theorem 3.1. For any finite $t_{0}>0$ and any $\phi \in H,\left\|T^{N}(t) \pi^{N} \phi-\pi^{N} T(t) \phi\right\| \rightarrow 0$ as $N \rightarrow \infty$ uniformly in $t \in\left[0, t_{0}\right]$.

\section{Proof.}

The proof is consequence of the above Lemmas and Trotter-Kato Theorem.

Theorem 3.2. For any finite $t_{0}>0$, the semi-discrete solutions of equation (3.1) converges in $\|\cdot\|$ to the unique solution of (1.1), uniformly on the interval $\left[0, t_{0}\right]$ 
Proof. Note that the solution of the semidiscrete equation (3.1) satisfies

$$
\rho^{N}(t)=T^{N}(t) \pi^{N} \rho_{0}+\int_{0}^{t} T^{N}(t-s) \pi^{N} F\left(\rho^{N}(s)\right) d s .
$$

Subtracting this expression from the original mild equation, we see that an application of Theorem 3.1. and Gronwall inequality will establish the result (for similar results see Ackleh et. al. (1994a)).

\section{Numerical Results}

In this section we present some numerical simulations. In the first example, we give some basic computations that explore the accuracy of the method. The second and third examples illustrate the complementary effects of growth and coagulation in the model.

Testing the method

Since we do not know of any closed form solution for equation (1.1) we used the following technique to test our method. We choose a solution and we make our equation obey this solution by adding a forcing term so we will instead be solving the following equation

$$
\left\{\begin{array}{l}
\frac{\partial \rho}{\partial t}+\frac{\partial(g \rho)}{\partial x}=F(\rho)+f(t, x) \\
g\left(x_{0}\right) \rho\left(t, x_{0}\right)=K(\rho) \\
\rho(0, x)=\rho_{0}(x) .
\end{array}\right.
$$


Assuming our solution is $e^{-t} \sin \pi x$ and choosing the following functions for our parameters

$$
\begin{gathered}
x_{0}=0, x_{1}=1, g(x)=1-x, q(x)=0 \\
\beta(x, y)=\left\{\begin{array}{l}
1-x-y \text { for } x+y \leq 1 \\
0 \text { otherwise }
\end{array}\right.
\end{gathered}
$$

We find that our function

$$
\begin{aligned}
f(t, x)= & -2 \sin \pi x+\pi(1-x) \cos \pi x+\frac{3}{4 \pi}(1-x) \sin \pi x \\
& +\frac{1}{4} x(1-x) \cos \pi x+\frac{1}{\pi^{2}} \sin \pi x \sin \pi(x-1)
\end{aligned}
$$

In all our simulation we have used a first order discretization in time. Fig. 1 represents the $L^{\infty}$ error. We note that the convergence appears to be of first order, which is what we expect since we have used first order approximation both in time and space.

\section{Application to biology}

We note that Ackleh and Hallam (1994) showed that densities of large aggregate sizes have been predicted to be lower in their model then actual experimental values. They argued that one solution for this problem is that experimental contact efficiencies used in there model simulation are lower than actual ones and hence they have used inverse method to estimate a new contact effiencies which have resulted in much better fit for large aggregates.

We will now show, using numerical simulations, how such low densities can be considerably higher by incorporating a growth term for aggregates. To concentrate 
our efforts on growth versus aggregation we will assume that the reproduction function $q(x)=0$. In order not give the growth too much weight we choose the function $g(x)=0.1(1-x)$ and to give aggregation more weight than the former example we muliply $\beta(x, y)$ by a factor of 10 to get

$$
\beta(x, y)=\left\{\begin{array}{l}
10(1-x-y) \text { for } x+y \leq 1 \\
0 \quad \text { otherwise }
\end{array}\right.
$$

In Fig. 2a we present the density distribution for the model when $g(x)=0$ and in Fig. $2 b$ the density of distribution for the model is presented using the above choice of $g$. Fig. 2c presents the difference at the final time for these two distributions. Notice that with such a relatively low growth term the densities of large aggregates are almost twice as high as when no growth is incorporated.

In the above example we showed (using a somewhat nonrealistic function of coagulation kernel) that including a growth term can solve the problem of predicting low densities for large aggregates without using different contact efficienies than the ones in the literature. We will now present another example using a (real) stickiness function from Ackleh et. al. (1994b) that has the form

$$
\beta(x, y)=\left\{\begin{array}{l}
c_{1}\left(d_{x}+d_{y}\right)^{3} E C_{x, y}+(\pi / 4) *\left(d_{x}+d_{y}\right)^{2}\left|w_{x}-w_{y}\right| E C_{x, y} \text { for } x+y \leq 1 \\
0 \quad \text { otherwise }
\end{array}\right.
$$

Where

$$
E C_{x, y}=\left(d_{\min } / d_{\max }\right)^{p} /\left(1+d_{\min } / d_{\max }\right)^{p}
$$

is the contact efficiency and $d_{\min }=\min \left(d_{x}, d_{y}\right)$, where $d_{x}$ is the diameter of aggregate of volume $x$ and similarly $d_{y}$ is the diameter of aggregate of volume $y$, and $d_{\max }$ is similarly defined. 
Our goal is to present a difference between the final distributions of aggregates when simulating the following:

1) the system with $g=0$ (no growth) and using the contact efficiency function developed by McCave (1984) which used a surface area contact efficiency, i.e.. $p=2$ in $(4.2)$.

2) the system with $g=0$ and using the contact efficiency estimated by Ackleh and Hallam (1994) which is of the form (4.2) with $p=1.2$.

$3)$ the system with a growth function $g=(1-s)(s+.01)$ and the contact efficiency developed by McCave (1984).

We again assumed that $q(x)=0$ and we chose an initial distribution of aggregates that relates to the densities at a peak of the bloom. All simulations were for three days to check the difference in final distribution of aggregates right around the bloom, which is of course the most important period for high aggregation (Hill, 1992). Fig3a represents the distributions of aggregates for the above three cases and proves the point that both processes,i.e contact efficiency and growth, are important to improve the prediction for large aggregate densities. Fig3b magnifies Fig3a for the final distributions for aggregates of volumes $x>0.6 \mathrm{~mm}^{3}$, and show that the results are even higher than the ones where the contact efficiency estimated by Ackleh and Hallam (1994) is used which could improve the fit by Ackleh and Hallam which still presented lower densities for large aggregats.

The idea behind such a result is very simple. The growth term shifts aggregates with small sizes and usually much higher densities into larger ones, but then since the real stickiness function used depends on the volume those aggregates now will 
tend to aggregate much faster into large ones than if they would have stayed at their original size (no growth term). Hence growth and aggregation are processes that enhance each other.

Fig. 1. $L_{\infty}$ error 
Fig. 2a. The density distribution using $g(x)=0$. 
Fig. $\mathbf{2 b}$ The density distribution using $g(x)=0.1(1-x)$. 
Fig. 2c The difference in density distribution at the final time between Fig. 2a and Fig. $2 b$. 
Fig. 3a The density distribution at the final time for the case (1) $g=0, p=2(2)$ $g=0, p=1.2$ and $(3) g=(1-s)(s+.01), p=2$. 
Fig. 3b The same as Fig3a for aggregates with volume $x>0.6 \mathrm{~mm}^{3}$.

\section{Concluding remarks.}

We have examined an algal dynamics model that includes the effects of coag-

ulation and growth. Numerical studies indicate that even very small aggregate growth can have a major impact on the formation of large aggregates. While these 
computations illustrate the relative effects, they do not allow us to conclude from experimental data what levels of growth and stickiness to choose.

In order to make definitive quantitative comparisons, one must develop inverse methods for estimating both the growth and stickiness functions. Quantities that have been obtained in experimental settings are values of the size densities (Alldrege and Gotschalk, 1988: Riebesell, 1991; Jackson, 1994). Methods for estimating the stickiness function have been developed (Ackleh et al. 1994; Kiorboe et al., 1990) within the context of a purely coagulation model. In models which include aggregate growth, it is typically assumed (Jackson, 1990; Jackson and Lochmann, 1991) that only single cells (non-aggregated) divide and cells in aggregates do not or (Ackleh et. al., 1994) that cells that are born in aggregates fall off the aggregate and join the single cell population. The reason behind such assumptions is the tremendous difficulty in trying to model a growth function of an aggregate since it is composed of many individual cells, each of which might have it's own growth rate. Such assumptions may underestimate the importance of the biological processes in comparison to the physical processes of the aggregation and sinking, and in future studies we plan to develop parameter estimation techniques that will aid in such comparisons and give us an idea of possbile functions that might model the growth term. 


\section{REFRENCES}

1. Ackleh, A. S., Fitzpatrick, B. G., Hallam T. G.: Approximation and parameter estimation for algal aggregation models. Mathematical Models and Methods in Applied Sciences 4, 291-311 (1994a).

2. Ackleh, A. S., Hallam T. G.: Estimation of sticking and contact efficiencies in phytoplankton aggregation: The 1993 SIGMA tank experiment. submitted (1994).

3. Ackleh, A. S., Hallam T.G., Smith, W. O.: Influences of aggregation and grazing on phytoplankton dynamics and fluxes: An individual-based Modeling approach. Nonlinear World (to appear, 1994b).

4. Banks, H. T., Kappel, F.: Transformation semigroups and $L^{1}$-approximation for size structured population models. Semigroup Forum, 38, 141-155 (1989).

5. Banks, H. T., Kappel, F., Wang, C.: Weak solutions and differentiability for size structured population models. International Series of Numerical Mathematics, 100, 35-50 (1991).

6. Banks, H. T., Kunisch, K. Estimation techniques for distributed parameter systems. Birkhauser. PP 315 (1989).

7. Hill, P. S.: Reconciling aggregation theory with observed vertical fluxes following phytoplankton blooms. Journal of Geophysical Research, 97,2295$2308(1992)$. 
8. Jackson, G.: SIGMA Data Report 1. Santa Barbara Tank Experiment (1994).

9. Jackson, G.: A model of formation of marine algal flocs by physical coagulation processes. Deep-Sea Research, 37, 1197-1211 (1990).

10. Jackson, G., Lochmann, S.: Effect of coagulation on nutrient and light limitation of an algal bloom, em Limnology and Oceonography, 37, 77-89 (1992).

11. Kiorboe, T. , Anderson, K. P., Dam, H. G.: Coagulation efficiency and aggregate formation in marine phytoplankton. Marine Biology, 107, 235$245(1990)$.

12. McCave, I. N.: Size spectra and aggregation of suspended particles in the deep ocean. Deep-Sea Research, 31, 329-352 (1984).

13. Pazy, A.: Semigroup of Linear Operators and Applications to Partial Differential Equations. pp 279. 